\title{
Effects of humic substances on the aqueous stability of cerium dioxide nanoparticles and their toxicity to aquatic organisms
}

\author{
Qi Yu ${ }^{\text {a }}$, Zhuang Wang b,*, Yujia Zhai ${ }^{\text {a }}$, Fan Zhang ${ }^{\text {a }}$, Martina G. Vijver ${ }^{\text {a }}$, Willie J.G.M. Peijnenburg ${ }^{\text {a,c }}$ \\ a Institute of Environmental Sciences (CML), Leiden University, Leiden 2300, RA, the Netherlands

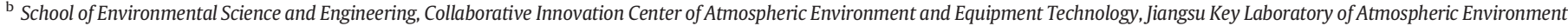 \\ Monitoring and Pollution Control, Nanjing University of Information Science and Technology, Nanjing 210044, PR China \\ ${ }^{c}$ Centre for Safety of Substances and Products, National Institute of Public Health and the Environment (RIVM), Bilthoven 3720 BA, the Netherlands
}

\section{H I G H L I G H T S}

- Exposure characteristics of test species affected the toxicity of $\mathrm{nCeO}_{2}$.

- Different concentrations of humic substances alleviated $\mathrm{nCeO}_{2}$ toxicity to R. subcapitata and to C. sphaericus.

- The joint effects of humic substances and $\mathrm{nCeO}_{2}$ were additive and synergistic to $D$. rerio.

\section{G R A P H I C A L A B S T R A C T}

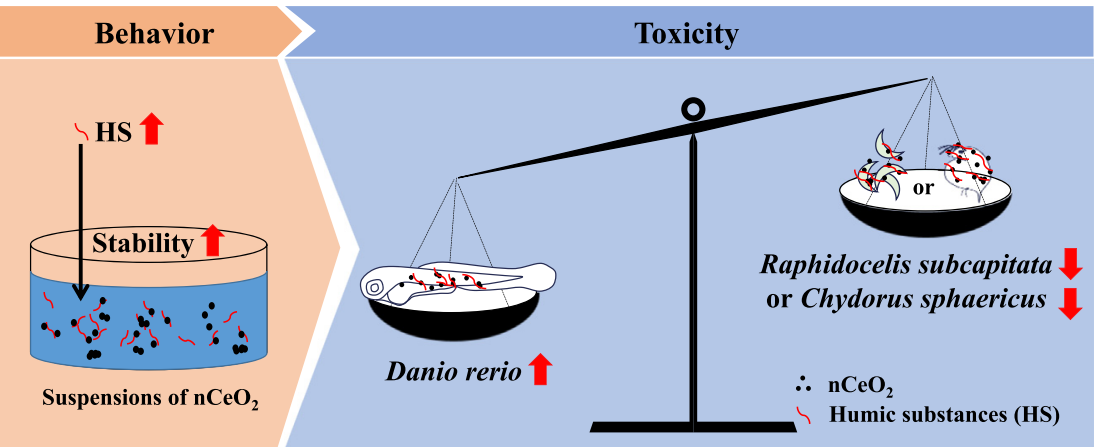

\begin{abstract}
A B S T R A C T
The impacts of humic substances $(\mathrm{HS})$ on the aquatic stability and toxicity of nano-cerium dioxide $\left(\mathrm{nCeO}_{2}\right)$ to three organisms with different exposure characteristics were investigated. Addition of HS to suspensions of $\mathrm{nCeO}_{2}$ lowered the surface zeta potential of the particles, reduced their hydrodynamic size, and increased the energy barrier as indicated by the total potential energy profile. This resulted in a more stable suspension compared to suspensions without HS added. Moreover, a higher concentration of HS further stabilized $\mathrm{nCeO}_{2}$ in the suspension. Acute toxicity of the suspensions to the unicellular green alga Raphidocelis subcapitata and to the crustacean Chydorus sphaericus was lower as compared to exposure without $\mathrm{HS}$ added. The acute toxicity of $\mathrm{nCeO}_{2}$ suspensions to the zebrafish (Danio rerio) eleutheroembryo was on the other hand significantly enhanced (additive and synergistic) upon increasing HS concentration. Our findings emphasize that HS is important to stabilize the nanosuspensions and that its impact on $\mathrm{nCeO}_{2}$ toxicity differs across different aquatic organisms. Emphasizing the exposure characteristics of each of the organisms selected from the trophic levels can explain how particle stability impacts particle toxicity.
\end{abstract}

(c) 2021 Elsevier B.V. All rights reserved.

\section{Introduction}

The prospects of engineered nanoparticles (ENPs) of different sizes, shapes, and material properties in a number of applications have

\footnotetext{
* Corresponding author.

E-mail address: zhuang.wang@nuist.edu.cn (Z. Wang).
}

progressed rapidly (Guinée et al., 2017; Martínez et al., 2020), although the market benefits brought about by ENPs have also created some concerns of their possible effects on human health and environmental safety (Savolainen et al., 2013; Baun et al., 2017; Deng et al., 2017). Many studies emphasized the challenging relationship between typical ENPs features such as shape, size, modifications and the abiotic factors of the environment such as $\mathrm{pH}$, divalent cation ions, dissolved organic 
carbon (Lu et al., 2017; Liu et al., 2018; Yu et al., 2018; Singh et al., 2021). Understanding how ENPs interact with the environment can assist in predicting the fate and effect of ENPs and may provide a basis for their ecological risk assessment.

Nano-cerium dioxide $\left(\mathrm{nCeO}_{2}\right)$ is increasingly being applied in fuel additives and polishing agents (Collin et al., 2014). To date, the estimated production of $\mathrm{nCeO}_{2}$ is around 1000 tons/year and it has become one of the most produced ENPs in the world (Piccino et al., 2012). Upon their release into the aquatic environment, $\mathrm{nCeO}_{2}$ may provoke adverse effects to various organisms (Rundle et al., 2016; Taylor et al., 2016; Kosak Née Röhder et al., 2018; Wang and Nowack, 2018; Correia et al., 2020).

Natural organic matter (NOM) is ubiquitous in every water and is known to affect the fate and toxicity of ENPs by modifying the surface properties (e.g., charge and hydrophobicity), subsequently affecting their stability as well as nanoparticle-cellular interactions (Van Hoecke et al., 2011; Loosli et al., 2013; Baalousha et al., 2018; Liu et al., 2020). Evidence was found that the presence of Suwannee River and Bihain NOM can stabilize $\mathrm{nCeO}_{2}$ in an algae growth medium (Quik et al., 2010). Moreover, Suwannee River NOM can alleviate the adverse effects of NPs on algal growth (Cerrillo et al., 2016). However, how the $\mathrm{nCeO}_{2}-\mathrm{NOM}$ interaction alters the toxicity of $\mathrm{nCeO}_{2}$ to aquatic organisms is not completely understood.

This work aimed to explore the impact of humic substances (HS) on the aqueous stability and subsequent toxicity of $\mathrm{nCeO}_{2}$ to different organisms. The aqueous stability of $\mathrm{nCeO}_{2}$ in the presence of $\mathrm{HS}$ was determined by measuring the zeta potential of suspensions and the sizes of the agglomerates formed, whereas suspension concentrations were measured in simulated natural environmental conditions. Short-term experiments were performed with aquatic organisms with different exposure characteristics, namely the microalga Raphidocelis subcapitata, the microcrustacean Chydorus sphaericus, and the fish Danio rerio. The different exposure characteristics of the organisms assist in understanding the actual exposure of aquatic organisms to nanoparticles and the effective interactions with nanoparticles. The starting hypotheses were:

$\mathbf{H O}_{\mathbf{1}}$. . Algal cells and HS will compete for binding with the nanoparticles. Thus, it is expected that the addition of $\mathrm{HS}$ to $\mathrm{nCeO}_{2}$ suspensions will diminish the toxicity of the suspensions to algae.

$\mathbf{H O}_{2}$. . The microcrustacean are bottom-feeders that graze on the bottom of the vessels. Stabilization of $\mathrm{nCeO}_{2}$ suspensions by $\mathrm{HS}$ decreases the toxicity to Chydoridae compared to relative instable $\mathrm{nCeO}_{2}$ suspensions without HS addition.

$\mathbf{H O}_{3}$. . Nanoparticle sedimentation increases the particle concentration at the bottom of the test well and that is where the zebrafish embryos reside. Accordingly, the embryos are exposed to $\mathrm{nCeO}_{2}$ at a higher extent when the $\mathrm{nCeO}_{2}$ is not stabilized. When hatched, the larvae swim around and mix the $\mathrm{nCeO}_{2}$ and thus higher toxicity is expected without HS added.

\section{Materials and methods}

\subsection{Test materials and medium}

$\mathrm{CeO}_{2}$ nanoparticles were supplied by Umicore Ltd. as powders. The particles were redispersed and stocked by milling into Milli-Q water as $10 \mathrm{wt} \%$ suspensions at $\mathrm{pH} 4$ containing nitric acid, as well as stored at $4{ }^{\circ} \mathrm{C}$ for later use. Moreover, the test suspensions were characterized immediately after their preparation. The primary particle size of $\mathrm{nCeO}_{2}$ was $20 \mathrm{~nm}$ and the specific surface area was $42 \mathrm{~m}^{2} / \mathrm{g}$ as determined by the manufacturer. The model HS purchased from J\&K Chemical, a humic acid sodium salt (50-60\% as humic acid, CAS: 68131-04-4), was selected to mimic natural organic matter (NOM). The humic acid sodium salt acts as a proxy for humic-like substances that were also identified as a major component of NOM. The exposure medium was defined to be the Dutch standard (DSW) (Hermsen et al., 2011) consisting of $\mathrm{NaHCO}_{3}(1.19 \mathrm{mM}), \mathrm{KHCO}_{3}(0.20 \mathrm{mM}), \mathrm{CaCl}_{2}(1.36 \mathrm{mM})$, and $\mathrm{MgSO}_{4}$ $(0.73 \mathrm{mM})$ with a $\mathrm{pH}$ adjustment of $8.0 \pm 0.2$.

\subsection{Treatments and concentrations tested}

Drop-wise addition of the $\mathrm{nCeO}_{2}$ stock suspensions into the DSW was carried out to prepare the different test dosages of the nanoparticles, which were $0.5,1,5,10,25,50,100 \mathrm{mg} / \mathrm{L}$. Moreover, the test suspensions of the $\mathrm{nCeO}_{2}$ were stirred for $24 \mathrm{~h}$ in the dark. A HS stock solution was prepared by dissolving $100 \mathrm{mg} / \mathrm{L}$ by stirring for $24 \mathrm{~h}$ at room temperature and filtering over a $0.20 \mu \mathrm{m}$ nylon membrane filter prior to use. The actual HS concentrations of suspensions of nominal $0.5,10$, and $40 \mathrm{mg} \mathrm{C} / \mathrm{L}$ in the DSW were measured by using a Thermo Hiper TOC analyzer. The $\mathrm{nCeO}_{2}$ suspensions in the presence of $\mathrm{HS}$ were prepared by adding the $\mathrm{nCeO}_{2}$ stock suspensions into the $\mathrm{HS}$ solutions. The suspensions of $\mathrm{nCeO}_{2}$ with $\mathrm{HS}$ were further stirred for $24 \mathrm{~h}$ in the dark. The $\mathrm{pH}$ values of all samples were adjusted to $8.0 \pm 0.2$ using a $1 \mathrm{~mol} / \mathrm{L} \mathrm{HCl}$ or $\mathrm{NaOH}$ solution.

\subsection{Physical and chemical analysis}

The physical and chemical measurements were performed on the 10 $\mathrm{mg} / \mathrm{L} \mathrm{nCeO}{ }_{2}$ suspension in glass flasks $(5 \mathrm{~cm}$ in diameter and $10 \mathrm{~cm}$ in height) with $100 \mathrm{~mL}$ under the same conditions as the toxicity testing for $0,24,48,72$, and $96 \mathrm{~h}$. Zeta potentials, mean (the average of all particles) particle diameters, and particle concentrations of suspensions in the absence and presence of HS were determined at the end of different intervals. The zeta potential was measured by a ZetaSizer (Nano series, Malvern Instruments) in triplicate. The particle size distribution of $\mathrm{nCeO}_{2}$ in the DSW was measured by a Nanosight LM20 system (NanoSight Ltd., Salisbury, UK) using Nanoparticle Tracking Analysis (NTA) (software version 1.5). From the classical Derjaguin-LandauVerwey-Overbeek (DLVO) theory (Cosgrove, 2005), the stability of a particle in the test medium was determined by simulating the total potential energy for interactions between the $\mathrm{CeO}_{2}$ particles in the absence and presence of HS. The detailed process and parameter settings of the DLVO calculations are described in the Supplementary Information. The total Ce concentration was determined by high resolution inductively coupled plasma mass spectroscopy (Element 2 HR-ICP-MS, Thermo, Bremen, Germany) after a $2 \mathrm{~h}$ hot open destruction treatment with 1 $\mathrm{mL}$ hydrogen peroxide $(9.8 \mathrm{~mol} / \mathrm{L})$ and $7 \mathrm{~mL}$ nitric acid $(14.4 \mathrm{~mol} / \mathrm{L})$ at $103^{\circ} \mathrm{C}$.

\subsection{Bioassays}

Toxicity of the $\mathrm{nCeO}_{2}$ in the absence and presence of $\mathrm{HS}$ to the test organisms was assessed by concentration-response experiments. For each batch of toxicity tests, a control consisting of the test medium was included to ensure that the observed effects were associated with exposure to the test compounds.

Microalga R. subcapitata from a continuous culture were suspended in DSW to a total volume of $3 \mathrm{~mL}$ in glass vials, to obtain a final density of $1 \times 10^{6}$ cells $/ \mathrm{mL}$. The inhibition of $4.5 \mathrm{~h}$ photosynthetic efficiency was determined for the algae using a pulse-amplitude modulation fluorometer (Wang et al., 2012). The algae were exposed to various initial concentrations $(0.5,1,5,10,25,50$, and $100 \mathrm{mg} / \mathrm{L})$ of the $\mathrm{nCeO}_{2}$ suspensions in the absence and presence of $\mathrm{HS}(0.5,10$, and $40 \mathrm{mg} \mathrm{C} / \mathrm{L})$, as well as the HS alone $(0.5,10$, and $40 \mathrm{mg} \mathrm{C/L})$.

The $48 \mathrm{~h}$ acute immobilization tests with $C$. sphaericus followed the protocol of the Chydotox toxicity test developed in the National Institute of Public Health and the Environment (Netherlands) (Verweij et al., 2009). Twenty neonates ( $<24 \mathrm{~h}$ ) at each exposure concentration, divided into four batches of five animals each, were added to a single droplet of DSW with a diameter of approximately $5 \mathrm{~mm}$ in the glass 
vial. $250 \mu \mathrm{L}$ test solution was added into each vial. The vials were covered with a crimp cap to prevent evaporation and incubated for $48 \mathrm{~h}$ in a climate room at $20^{\circ} \mathrm{C}$ and a light: dark regime of $16: 8 \mathrm{~h}$.

Crustaceans $C$. sphaericus were exposed to various initial concentrations $(0.5,1,5,10,25,50$, and $100 \mathrm{mg} / \mathrm{L})$ of the $\mathrm{nCeO}_{2}$ suspensions in the absence and presence of $\mathrm{HS}(0.5,10$, and $40 \mathrm{mg} \mathrm{C} / \mathrm{L})$, as well as the HS alone $(0.5,10$, and $40 \mathrm{mg} \mathrm{C} / \mathrm{L})$. After $48 \mathrm{~h}$, the vials were placed under a reverse dissecting microscope and the immobility was determined by activation of $C$. sphaericus by slightly shaking the vial and monitoring them for $30 \mathrm{~s}$.

An early life stage test with zebrafish (D. rerio) embryos was performed in accordance with the procedure described by Lammer et al. (2009). Each exposure concentration and control in 3 independent experiments was tested with 10 eggs/embryos (4-64 cell stage) from 20 vital and fertilized eggs, and the selected eggs were transferred into 24-well cell culture plates with $2 \mathrm{~mL}$ freshly prepared control medium or test suspensions. Embryos were incubated at $26 \pm 1{ }^{\circ} \mathrm{C}$ for $96 \mathrm{~h}$ and were exposed to three $\mathrm{nCeO}_{2}$ exposure concentrations $(1,10$, and $100 \mathrm{mg} / \mathrm{L}$ ) in the absence and presence of HS (10 and $40 \mathrm{mg} \mathrm{C} / \mathrm{L}$ ), as well as the $\mathrm{HS}$ alone ( 10 and $40 \mathrm{mg} \mathrm{C/L}$ ). The development of zebrafish embryos was microscopically screened daily. Lethality as well as sub-lethal developmental morphology and teratogenicity toxicological endpoints (Hermsen et al., 2011) were scored after 96 h (Supplementary Information, Table S1). Embryos were scored according to the classifications given by King-Heiden et al. (2009) as (0) no toxic response, (1) one or two toxic endpoints, (2) two or three toxic endpoints, (3) more than three toxic endpoints, and (4) dead. The choice of the scores depends on the severity of the toxicity endpoint. In order to avoid overestimating the toxicity of the test materials, a lower score was selected if a certain toxicity endpoint of an embryo was slightly damaged.

\subsection{Toxicity evaluating of $n \mathrm{CeO}_{2}$ in the presence of $\mathrm{HS}$}

$\mathrm{nCeO}_{2}$ with the addition of $\mathrm{HS}$ can be regarded as a binary mixture system. The observed mixture toxicity effect, $M E_{\mathrm{obs}}$, determined in the toxicity testing was compared with the theoretically predicted mixture toxicity effect, $M E_{\text {pre, }}$, calculated using a probability theory based model (Hadjispyrou et al., 2001):

$M E_{\mathrm{pre}}=E_{\mathrm{nCeO} 2}+E_{\mathrm{HS}}-\left(E_{\mathrm{nCeO} 2} \cdot E_{\mathrm{HS}} / 100\right)$

where $E_{\mathrm{nCeO} 2}$ is the single toxicity effect of $\mathrm{nCeO}_{2}$ and $E_{\mathrm{HS}}$ is the single toxicity effect of $\mathrm{HS}$.

The result was applied to reflect a synergistic or antagonistic effect if $M E_{\text {obs }}$ was significantly higher or lower than $M E_{\text {pre, }}$ respectively. On the contrary, the interaction of the binary mixture was considered as an additive effect only if there was no significant difference between $M E_{\text {obs }}$ and $M E_{\text {pre }}$. The presented method was applied to predict the toxicity of the studied systems to $D$. rerio, which is due to the fact that there was no observed toxicity to the algae and to the cladoceran induced by the HS studied.

All values were reported as mean \pm standard deviation (SD). SD of the mean was calculated from parallel experiments. Statistically significant differences between test treatments in the present study were determined by the Student's $t$-test. The significance levels $p<0.05, p<$ 0.01 , and $p<0.001$ were used.

\section{Results and discussion}

\subsection{Effects of $\mathrm{HS}$ on aqueous stability of $n \mathrm{CeO}_{2}$}

The variation of the zeta potential values of $\mathrm{nCeO}_{2}$ with the $\mathrm{HS}$ concentrations and with the time of incubation is shown in Fig. 1A. The zeta potential values of the $\mathrm{nCeO}_{2}$ were found to be negative and changed slightly over time. Furthermore, the zeta potential values were more negative when the HS were present compared with the zeta potential values when the HS were absent, implying that the HS can influence electrokinetic properties, causing an increased nanoparticle surface charge. The HS effect on the particle zeta potential depended on the HS concentrations, irrespectively of the time of incubation.

Observed changes in the mean particle diameters with the HS concentrations over the experimental duration in the absence and presence of $\mathrm{HS}$ are presented in Fig. $1 \mathrm{~B}$. The $\mathrm{nCeO}_{2}$ particles immediately agglomerated in DSW-medium at an initial mean particle diameters size of 218 $\mathrm{nm}$, which is a tenfold higher than the primary size $(20 \mathrm{~nm})$. This implies that the $\mathrm{nCeO}_{2}$ particles were agglomerated. Upon increasing $\mathrm{HS}$ concentrations, the mean particle diameters of the $\mathrm{nCeO}_{2}$ agglomerates decreased. This may be explained because the HS acts as a polyelectrolyte or surfactant, thus providing electrostatic repulsive forces of the particles (Baalousha et al., 2008; Domingos et al., 2009). This stabilization of the particles by HS and subsequent decrease of the mean particle diameters of the agglomerates, was detectable regardless of exposure time.

On the basis of the DLVO theory (Cosgrove, 2005), the total potential energy profiles for the $\mathrm{nCeO}_{2}$ colloids with nanoparticle concentrations of $10 \mathrm{mg} / \mathrm{L}$ were calculated (Fig. 2). The magnitudes of the primary maximum of the $\mathrm{nCeO}_{2}$ colloids in the presence of the different concentrations of the HS follow the order: $\mathrm{nCeO}_{2}+10 \mathrm{mg} \mathrm{C} / \mathrm{L} \mathrm{HS}\left(19.1 \mathrm{~K}_{\mathrm{B}} \mathrm{T}\right)$ is approximately equal to $\mathrm{nCeO}_{2}+40 \mathrm{mg} \mathrm{C} / \mathrm{L} \mathrm{HS}\left(18.9 \mathrm{~K}_{\mathrm{B}} \mathrm{T}\right)>\mathrm{nCeO}_{2}+$ $0.5 \mathrm{mg} \mathrm{C} / \mathrm{L} \mathrm{HS}\left(9.8 \mathrm{~K}_{\mathrm{B}} \mathrm{T}\right)>\mathrm{nCeO}_{2}\left(6.6 \mathrm{~K}_{\mathrm{B}} \mathrm{T}\right)$, implying that the stability of the colloids studied follows the order: $\mathrm{nCeO}_{2}+10 \mathrm{mg} \mathrm{C} / \mathrm{L} \mathrm{HS}$ is
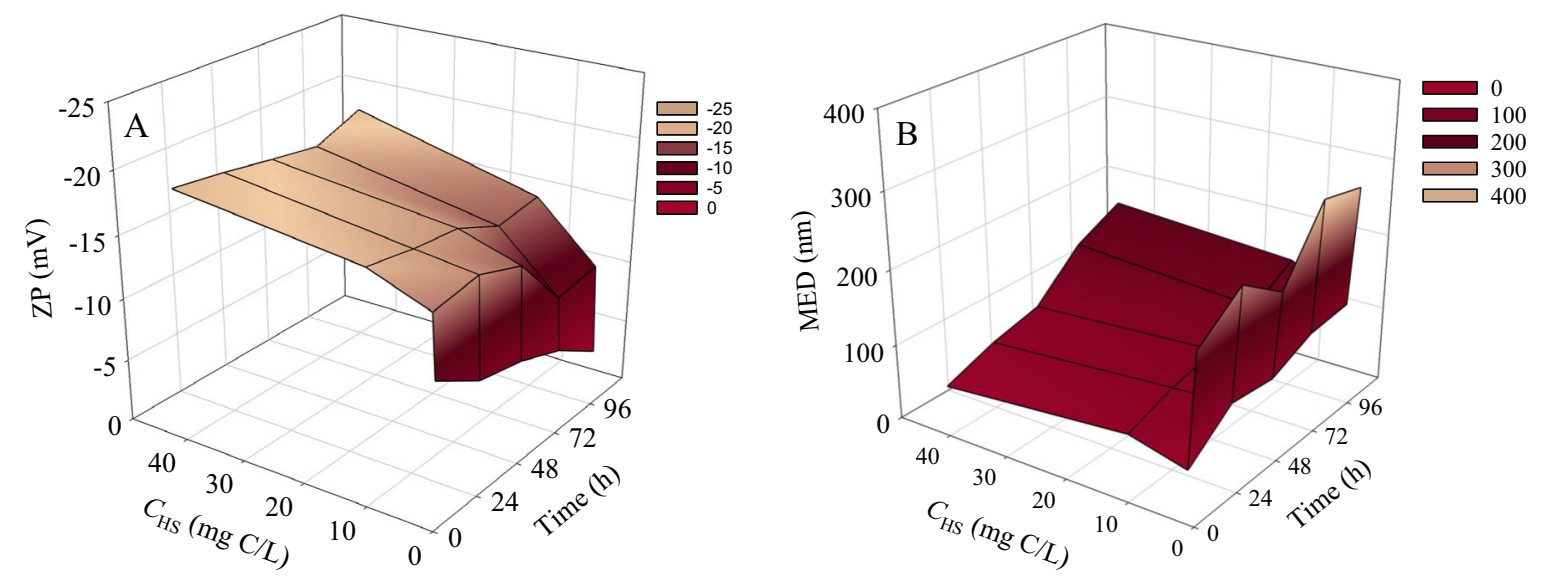

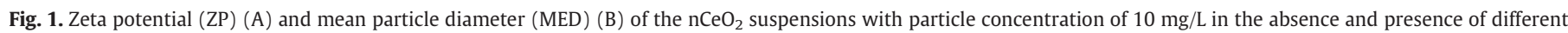
concentrations of $\mathrm{HS}\left(C_{\mathrm{HS}}\right)(0.5,10$, and $40 \mathrm{mg} \mathrm{C} / \mathrm{L})(\mathrm{pH}=8.0)$. Results are expressed as mean $(n=3)$. 


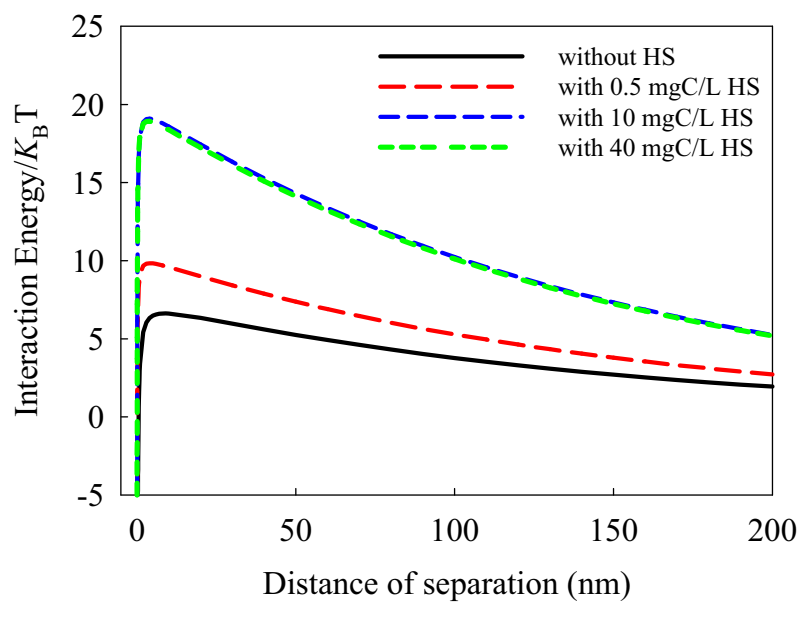

Fig. 2. Total potential energy curves for the $\mathrm{nCeO}_{2}$ with particle concentration of $10 \mathrm{mg} / \mathrm{L}$ in the presence of $\mathrm{HS}$ of $0.5,10$, and $40 \mathrm{mg} \mathrm{C} / \mathrm{L}$.

approximately equal to $\mathrm{nCeO}_{2}+40 \mathrm{mg} \mathrm{C} / \mathrm{LHS}>\mathrm{nCeO}_{2}+0.5 \mathrm{mg} \mathrm{C} / \mathrm{LHS}$ $>\mathrm{nCeO}_{2}$. It is the primary maximum in the total energy which provides the mechanism for the stability of the charged colloidal particles. As two particles approach each other, they must collide with sufficient energy to overcome a barrier provided by the primary maximum. Thus, the larger the barrier, the longer the system will remain stable. In the present study, we also observed that the stability of the binary systems of $\mathrm{nCeO}_{2}+10 \mathrm{mg} \mathrm{C} / \mathrm{L}$ HS is approximately equal to the stability of the combination of $\mathrm{nCeO}_{2}+40 \mathrm{mg} \mathrm{C} / \mathrm{L}$ HS. This also implies that only a part of the HS at the highest concentrations ( $40 \mathrm{mg} \mathrm{C} / \mathrm{L} \mathrm{HS}$ ) could be actually adsorbed.

The stability of $\mathrm{nCeO}_{2}$ in suspensions depends on the total potential energy of interaction between colloidal particles according to the DLVO theory. In the present study, the steric stabilization induced by HS was not considered in calculating the interaction energy between the colloidal particles as the thickness of the surface coating layer was found to be very small $(\leq 0.8 \mathrm{~nm})$ (Baalousha et al., 2008). Moreover, the studied HS analogue is a small molecule (MW: $226.14 \mathrm{~g} / \mathrm{mol}$ ) without any significant spatial extent and $\mathrm{nCeO}_{2}$ is thus considered to be a hard sphere. Although these assumptions and DLVO calculations are simplistic, they are indicative of the interaction forces between the nanoparticles and therefore the aggregation mechanisms (Baalousha, 2009).

Fig. 3A shows the variation of the ratios of the particle concentrations of suspensions to the initial particle concentration of $\mathrm{nCeO}_{2}$ in the absence and presence of HS over time. In the first $24 \mathrm{~h}$ this decline was steep and similar for all treatments. In the time span of $24 \mathrm{~h}$ to $96 \mathrm{~h}$ this steep decline could only be observed in the treatment without HS addition. The treatments with HS addition showed a dosedependent stabilization of the $\mathrm{nCeO}_{2}$ suspensions. As shown in Fig. 3B, the $\mathrm{nCeO}_{2}$-HS interaction resulted in a co-sedimentation behavior of
$\mathrm{HS}$ and $\mathrm{nCeO}_{2}$. This evidence supports the adsorption of HS upon the surface of the particles. Over $96 \mathrm{~h}$, the amount of HS adsorbed on the particles was estimated to be approximately $0.1,1.2$, and $1.8 \mathrm{mg} \mathrm{C} / \mathrm{L}$ corresponding to the initial HS concentration of $0.5,10$, and $40 \mathrm{mg} \mathrm{C} / \mathrm{L}$, respectively.

\subsection{Effects of $\mathrm{HS}$ on aquatic toxicity of $n \mathrm{CeO}_{2}$}

Concentration-dependent effects obtained for $\mathrm{nCeO}_{2}$ in the presence of different initial HS concentrations for the algae and cladoceran are given in Fig. 4, and for the zebrafish embryos in Fig. 5A. Throughout the test duration the $R$. subcapitata cells are gentle shaken continuously on a shaking table, hence the algal exposure was mainly within the water column where the nanoparticles were as well, representing relative high chances for exposure. As shown in Fig. 4A, the addition of 10 and $40 \mathrm{mg} \mathrm{C} / \mathrm{L} \mathrm{HS}$ to the $\mathrm{nCeO}_{2}$ suspensions significantly inhibited the acute toxicity measured as photosynthetic activity to R. subcapitata ( $p$ $<0.05$ ). Note that no toxic effects on the algae were observed for the HS. This means that the presence of 10 or $40 \mathrm{mg} \mathrm{C} / \mathrm{L} \mathrm{HS}$ interfered with the interaction of $\mathrm{nCeO}_{2}$ with the algal cells and weakened the toxic effect. This interference might also be explained by the fact that the physicochemical analysis indicated adsorption of $\mathrm{HS}$ to the $\mathrm{nCeO}_{2}$ particles, which subsequently hinders the particles from directly interacting with algal cells. Van Hoecke et al. (2011) also concluded that decrease in algal toxicity might be due to a reduction in bioavailability of the particles when NOM is present. Cerrillo et al. (2016) found that $\mathrm{NOM}$ alleviated the adverse effects of $\mathrm{nCeO}_{2}$ on algal growth to some extent and suggested a 'camouflage' effect of $\mathrm{nCeO}_{2}$.

The Chydoridae species is commonly grazing on the bottom of a vessel and sedimentation would increase exposure in case of particle agglomeration and subsequently sedimentation. These processes were largely affected by the addition of HS, with treatments without HS addition yielding relative instable nanoparticle suspensions. The acute toxicity of the $\mathrm{nCeO}_{2}$ to $\mathrm{C}$. sphaericus (Fig. 4B) was mitigated when $\mathrm{HS}$ was present, particular for the concentration of $40 \mathrm{mg}$ C/L HS. The physicochemical analysis here also showed that $\mathrm{HS}$ stabilized the $\mathrm{nCeO}_{2}$ suspensions. Taking into account that $C$. sphaericus is a benthic cladoceran species and is likely to ingest sediment particles into the gut and adsorb them on to carapaces, the reduced toxicity to $C$. sphaericus might be explained by the fact that a lower amount of NPs were allowed to sediment and subsequently, a lower amount of particles was accumulated in the cladoceran when HS was present as compared to the situation in which HS were absent. The analysis for the variation of the initial particle concentration of $\mathrm{nCeO}_{2}$ in the absence and presence of HS over time (Fig. 3A) also supports the conclusion that relatively more particles were stabilized when HS were present during the exposure.

As shown in Fig. $5 \mathrm{~A}$, the toxicity of $\mathrm{nCeO}_{2}$ to the larvae of $D$. rerio in the presence of $40 \mathrm{mg}$ C/L HS was significantly enhanced in comparison with the toxicity of $\mathrm{nCeO}_{2}$ to the larvae in the absence of $\mathrm{HS}$. In addition, the toxicity of $10 \mathrm{mg} \mathrm{C} / \mathrm{L} \mathrm{HS}$ alone treatment was equivalent to the
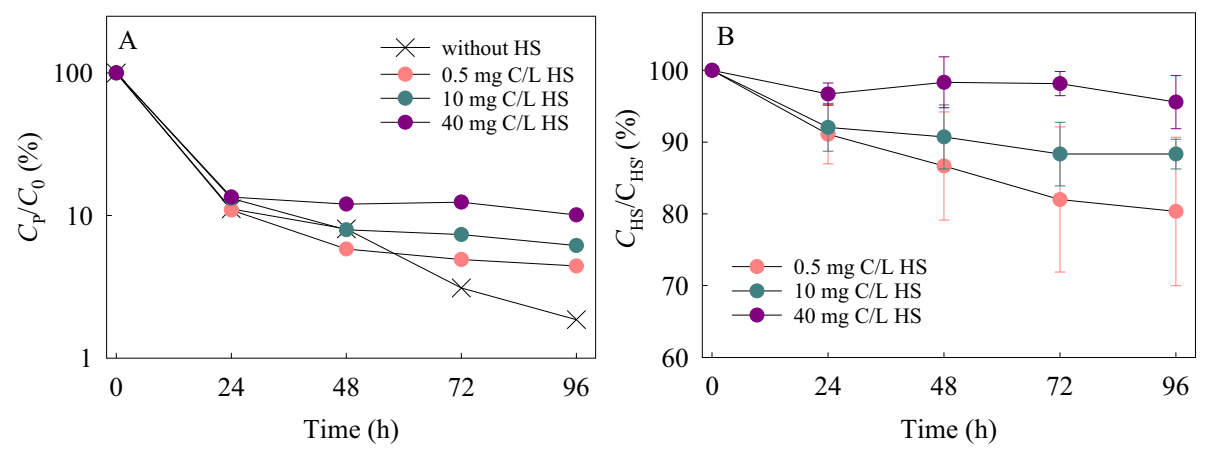

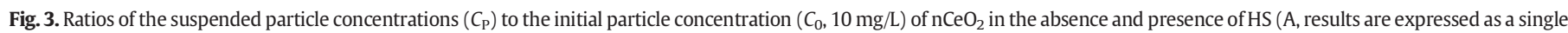

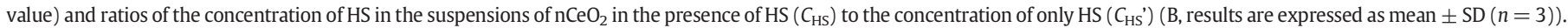



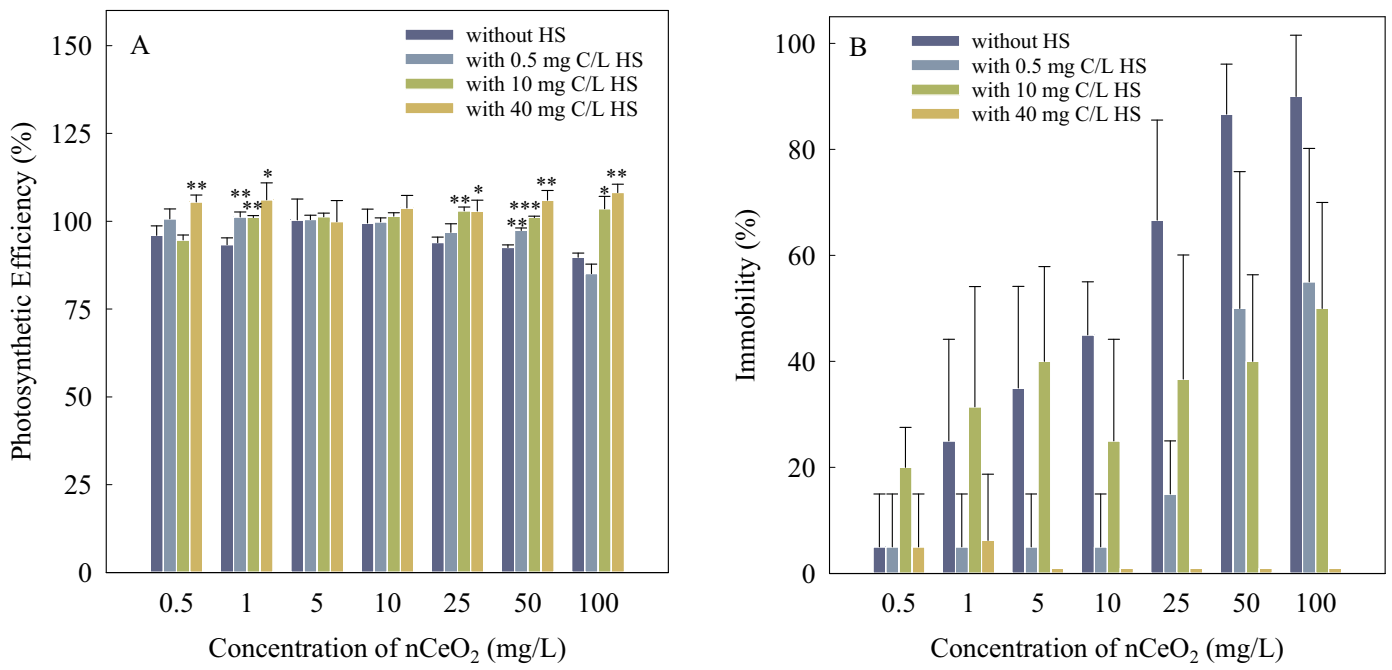

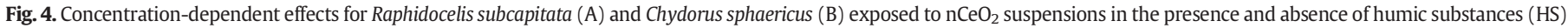
$\left(t\right.$-test ${ }^{*} p<0.05$ when comparing the treatments of $\mathrm{nCeO}_{2}$ without $\mathrm{HS}$ to $\mathrm{nCeO}_{2}$ with $\mathrm{HS}$ ). Results are expressed as mean $\pm \mathrm{SD}(n=3$ for $R$. subcapitata and $n=4$ for $C$. sphaericus).

toxicity of 1 and $10 \mathrm{mg} / \mathrm{L} \mathrm{nCeO}_{2}$ and significantly lower than $100 \mathrm{mg} / \mathrm{L}$ $\mathrm{nCeO}_{2}$. Thus, it can be concluded that concomitant exposure of the HS and $\mathrm{nCeO}_{2}$ caused the toxicity to $D$. rerio. The observed toxicity effects of the binary mixtures of the $\mathrm{HS}$ and $\mathrm{nCeO}_{2}$ were compared with their predicted toxicity (Fig. 5B), calculated using the result of individual toxic effects based on the eq. 1. No significant differences were observed for the cases 1 and $10 \mathrm{mg} / \mathrm{LCeO}_{2}+10 \mathrm{mg} \mathrm{C} / \mathrm{L} \mathrm{HS}$ between the results of the observation and prediction $(p<0.05)$, implying that these combinations showed additive action (observed toxicity similar to expected toxicity). The predicted mixture toxicity of the treatments 100 $\mathrm{mg} / \mathrm{L} \mathrm{CeO}_{2}+10 \mathrm{mg} \mathrm{C} / \mathrm{L} \mathrm{HS}$ and 1 and $10 \mathrm{mg} / \mathrm{L} \mathrm{CeO}_{2}+40 \mathrm{mg} \mathrm{C} / \mathrm{L} \mathrm{HS}$ was significantly greater than their observed mixture toxicity, indicating that these combinations had synergistic effects. In addition, the average value of the predicted toxicity of the binary mixture of $100 \mathrm{mg} / \mathrm{L} \mathrm{CeO}_{2}$ and $40 \mathrm{mg} \mathrm{C} / \mathrm{L} \mathrm{HS}$ was higher than the observed toxicity, although there was no significant difference between the observation and prediction.

The images of zebrafish larvae exposed to different concentrations of $\mathrm{nCeO}_{2}$ in the absence and presence of $\mathrm{HS}$ at $96 \mathrm{~h}$ depict the concentration-dependent malformations during development. Compared to the control (Fig. 5C), to the HS alone (Fig. 5D, no obvious anomalies), and to the individual $\mathrm{nCeO}_{2}$ (Fig. 5E, only scoliosis), the combination of $\mathrm{nCeO}_{2}$ and $40 \mathrm{mg} \mathrm{C} / \mathrm{L}$ HS induced more morphology and teratogenicity toxicity endpoints including pericardial oedema,
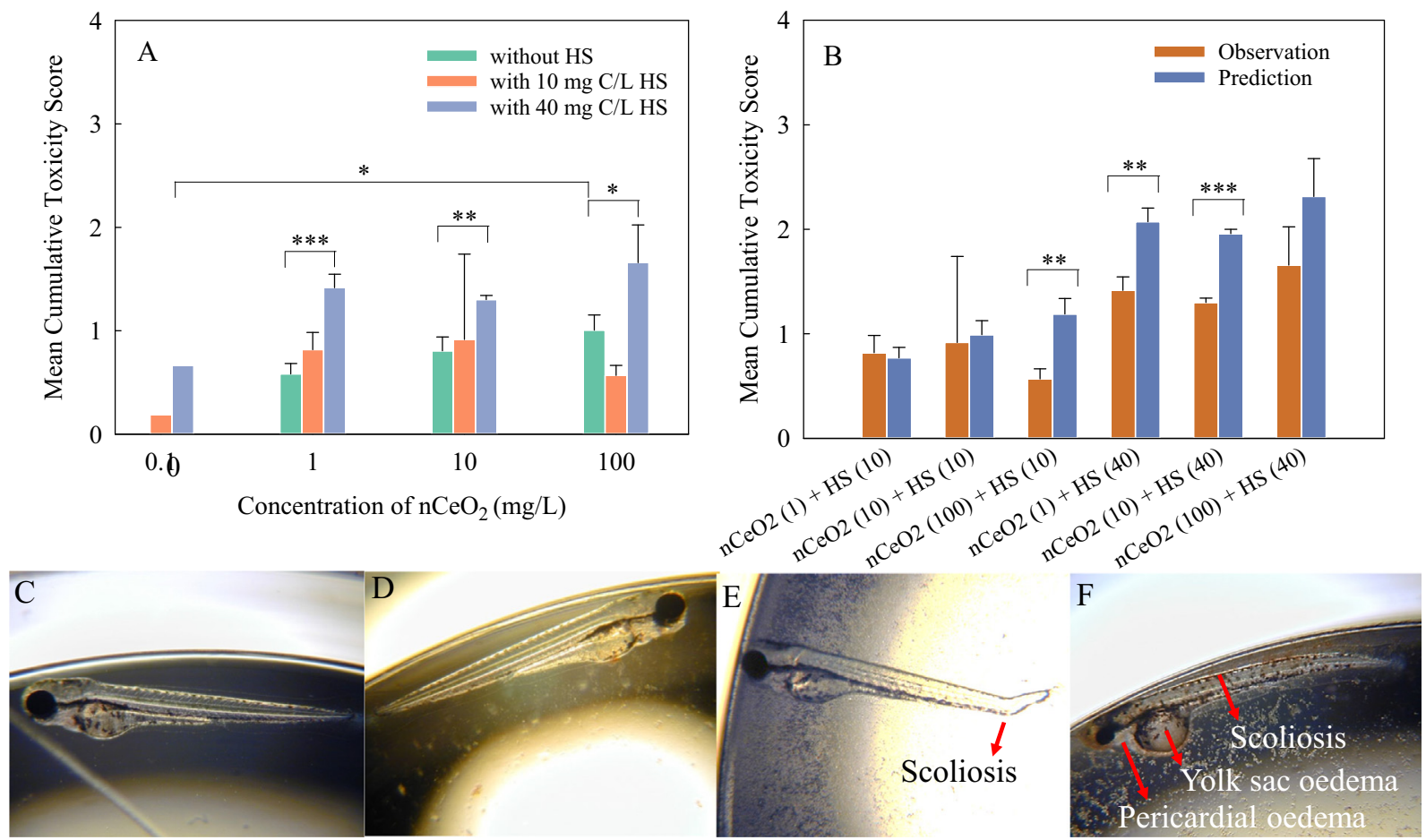

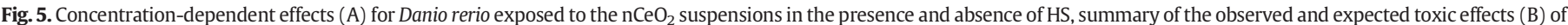

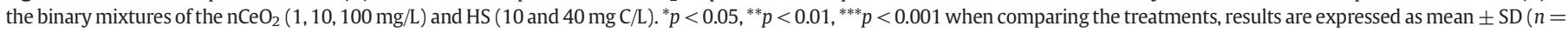

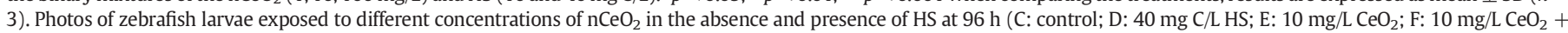
$40 \mathrm{mg} \mathrm{C} / \mathrm{L} \mathrm{HS}$ ). 
yolk sac oedema, and scoliosis (Fig. 5F). Here again, the presence of HS stabilized the particles of suspensions by decreasing their agglomerated MED size, implying that the zebrafish embryo would be exposed to the particles with lower size. This also means that the particles in suspensions with lower size induced more toxicity to the zebrafish larvae.

Thereupon, the zebrafish eggs with a chorion lie at the bottom of the test well. The chorion is considered as an effective barrier to protect the embryo from uptake of nanoparticles (Brun et al., 2018; Brinkmann et al., 2020). Due to the sedimentation of $\mathrm{nCeO}_{2}$, the zebrafish embryos were exposed to a higher extent to the nanoparticles. However, the chorion protected the zebrafish larvae against $\mathrm{nCeO}_{2}$ toxicity. After the hatching, the zebrafish larvae actively swam around in the water column and mixed with the nanoparticles. As aforementioned, the HS stabilized more nanoparticles than when the HS was absent. Consequently, the direct exposure of the zebrafish larvae to $\mathrm{nCeO}_{2}$ in the presence of $\mathrm{HS}$ was relatively higher than the direct exposure of the zebrafish larvae to $\mathrm{nCeO}_{2}$ in the absence of $\mathrm{HS}$, which could lead to a higher toxicity induced by $\mathrm{nCeO}_{2}$ when the HS was present. Furthermore, increasing the amount of the HS increased the severity of toxicity. Taken together, comparison analysis on the toxicity testing results suggests that the studied HS had a dual impact on the aquatic toxicity of $\mathrm{nCeO}_{2}$, depending on the exposure characteristics of the test species. Moreover, the HS concentration modulates its degree of influence on the toxicity.

\section{Conclusions}

Our results reveal how and to what extend the stability of $\mathrm{nCeO}_{2}$ was enhanced when HS was present in the aquatic medium at concentrations exceeding $0.5 \mathrm{mg} \mathrm{C} / \mathrm{L}$. Like expected by means of hypothesis $\mathrm{HO}_{1}$, the addition of $\mathrm{HS}$ mitigated the $\mathrm{nCeO}_{2}$ suspensions toxicity to algae $R$. subcapitata. The $\mathrm{HO}_{2}$ for the $C$. sphaericus species was accepted; $\mathrm{nCeO}_{2}$ suspensions stabilized by HS were less toxic as compared to relative instable suspensions of $\mathrm{nCeO}_{2}$ without $\mathrm{HS}$ added. Suspension of $\mathrm{nCeO}_{2}$ without $\mathrm{HS}$ added were more toxic to zebrafish larvae, which made us reject the $\mathrm{HO}_{3}$.

The extent and the effects of HS on the toxicity were associated with the concentration (ranging from 0.5 to $40 \mathrm{mg} \mathrm{C} / \mathrm{L}$ ) of HS added. Furthermore, the aqueous stability of $\mathrm{nCeO}_{2}$ and the aquatic species influenced the toxicity of the particles in the presence of HS. Our results are stepping stones towards improving the understanding the processes that determine the actual exposure of a suite of aquatic organisms to exposure media of different composition, mimicking to an increasing extend natural aquatic systems. Understanding the exposure characteristics of the organisms selected - explicitly considering where in the water column of the experimental test the organisms is most present will explain if stabilization of nanomaterials with HS or any other type of NOM will affect the toxicity of the nanomaterials.

\section{CRediT authorship contribution statement}

Qi Yu: Investigation, Data curation, Formal analysis, Writing - original draft, Visualization, Funding acquisition. Zhuang Wang: Conceptualization, Investigation, Methodology, Formal analysis, Validation, Writing - review \& editing. Yujia Zhai: Writing - review \& editing. Fan Zhang: Visualization, Writing - review \& editing. Martina G. Vijver: Conceptualization, Resources, Writing - review \& editing, Supervision, Funding acquisition, Project administration. Willie J.G.M. Peijnenburg: Conceptualization, Resources, Writing - review \& editing, Supervision, Funding acquisition, Project administration.

\section{Declaration of competing interest}

The authors declare that they have no known competing financial interests or personal relationships that could have appeared to influence the work reported in this paper.

\section{Acknowledgments}

The research described in this work was supported by the European Union's Horizon 2020 research and innovation programs "NanoinformaTIX" (grant number 814426), PATROLS (grant number $760813)$. Y.Q. greatly acknowledges the support from the China Scholarship Council (201706060195). We also thank the reviewers for their valuable comments on the manuscript.

\section{Appendix A. Supplementary data}

Supplementary data to this article can be found online at https://doi. org/10.1016/j.scitotenv.2021.146583.

\section{References}

Baalousha, M., 2009. Aggregation and disaggregation of iron oxide nanoparticles: influence of particle concentration, $\mathrm{pH}$ and natural organic matter. Sci. Total Environ. 407, 2093-2101.

Baalousha, M., Manciulea, A., Cumberland, S., Kendall, K., Lead, J.R., 2008. Aggregation and surface properties of iron oxide nanoparticles: influence of $\mathrm{pH}$ and natural organic matter. Environ. Toxicol. Chem. 27, 1875-1882.

Baalousha, M., Afshinnia, K., Guo, L., 2018. Natural organic matter composition determines the molecular nature of silver nanomaterial NOM-corona. Environ. Sci: Nano 5, 868-881.

Baun, A., Sayre, P., Steinhäuser, K.G., Rose, J., 2017. Regulatory relevant and reliable methods and data for determining the environmental fate of manufactured nanomaterials. NanoImpact 8, 1-10.

Brinkmann, B.W., Koch, B.E.V., Spaink, H.P., Peijnenburg, W.J.G.M., Vijver, M.G., 2020. Colonizing microbiota protect zebrafish larvae against silver nanoparticle toxicity. Nanotoxicology 14 (6), 725-739.

Brun, N.R., Koch, B.E.V., Varela, M., Peijnenburg, W.J.G.M., Spaink, H.P., Vijver, M.G., 2018. Nanoparticles induce dermal and intestinal innate immune system responses in zebrafish embryos. Environ Sci: Nano 5 (4), 904-916.

Cerrillo, C., Barandika, G., Igartua, A., Areitioaurtena, O., Mendoza, G., 2016. Towards the standardization of nanoecotoxicity testing: natural organic matter 'camouflages' the adverse effects of $\mathrm{TiO}_{2}$ and $\mathrm{CeO}_{2}$ nanoparticles on green microalgae. Sci. Total Environ. 543 (Pt A), 95-104.

Collin, B., Auffan, M., Johnson, A.C., Kaur, I., Keller, A.A., Lazareva, A., Lead, J.R., Ma, X., Merrifield, R.C., Svendsen, C., White, J.C., Unrine, J.M., 2014. Environmental release, fate and ecotoxicological effects of manufactured ceria nanomaterials. Environ Sci: Nano 1, 533-548.

Correia, A.T., Rodrigues, S., Ferreira-Martins, D., Nunes, A.C., Ribeiro, M.I., Antunes, S.C., 2020. Multi-biomarker approach to assess the acute effects of cerium dioxide nanoparticles in gills, liver and kidney of Oncorhynchus mykiss. Comp. Biochem. Physiol. C Toxicol. Pharmacol. 238, 108842.

Cosgrove, T., 2005. Colloid Science: Principles, Methods and Applications. Blackwell Publishing Ltd.

Deng, R., Lin, D., Zhu, L., Majumdar, S., White, J.C., Gardea-Torresdey, J.L., Xing, B., 2017. Nanoparticle interactions with co-existing contaminants: joint toxicity, bioaccumulation and risk. Nanotoxicology 11 (5), 591-612.

Domingos, R.F., Tufenkji, N., Wilkinson, K.J., 2009. Aggregation of titanium dioxide nanoparticles: role of a fulvic acid. Environ. Sci. Technol. 43, 1282-1286.

Guinée, J.B., Heijungs, R., Vijver, M.G., Peijnenburg, W.J.G.M., 2017. Setting the stage for debating the roles of risk assessment and life-cycle assessment of engineered nanomaterials. Nat. Nanotechnol. 12, 727-733.

Hadjispyrou, S., Kungolos, A., Anagnostopoulos, A., 2001. Toxicity, bioaccumulation and interactive effects of organotin, cadmium and chromium on Artemia franciscana. Ecotoxicol. Environ. Saf. 49, 179-186.

Hermsen, S.A.B., van den Brandhof, E.-J., van der Ven, L.T.M., Piersma, A.H., 2011. Relative embryotoxicity of two classes of chemicals in a modified zebrafish embryotoxicity test and comparison with their in vivo potencies. Toxicol. in Vitro 25, 745-753.

King-Heiden, T.C., Wiecinski, P.N., Mangham, A.N., Metz, K.M., Nesbit, D., Pedersen, J.A., Hamers, R.J., Heideman, W., Peterson, R.E., 2009. Quantum dot nanotoxicity assessment using the zebrafish embryo. Environ. Sci. Technol. 43, 1605-1611.

Kosak Née Röhder, L.A., Brandt, T., Sigg, L., Behra, R., 2018. Uptake and effects of cerium (III) and cerium oxide nanoparticles to Chlamydomonas reinhardtii. Aquat. Toxicol. 197, 41-46.

Lammer, E., Carr, G.J., Wendler, K., Rawlings, J.M., Belanger, S.E., Braunbeck, T., 2009. Is the fish embryo toxicity test (FET) with the zebrafish (Danio rerio) a potential alternative for the fish acute toxicity test? Comp. Biochem. Physiol. Part C Toxicol. Pharmcol. 149, 196-209.

Liu, J., Dai, C., Hu, Y., 2018. Aqueous aggregation behavior of citric acid coated magnetite nanoparticles: effects of pH, cations, anions, and humic acid. Environ. Res. 161, 49-60.

Liu, Y., Huang, Z., Zhou, J., Tang, J., Yang, C., Chen, C., Huang, W., Dang, Z., 2020. Influence of environmental and biological macromolecules on aggregation kinetics of nanoplastics in aquatic systems. Water Res. 186, 116316.

Loosli, F., Le Coustumer, P., Stoll, S., 2013. $\mathrm{TiO}_{2}$ nanoparticles aggregation and disaggregation in presence of alginate and Suwannee River humic acids, $\mathrm{pH}$ and concentration effects on nanoparticle stability. Water Res. 47, 6052-6063. 
Lu, K., Dong, S., Petersen, E.J., Niu, J., Chang, X., Wang, P., Lin, S., Gao, S., Mao, L., 2017. Biological uptake, distribution, and depuration of radio-labeled graphene in adult zebrafish: effects of graphene size and natural organic matter. ACS Nano 11, 2872-2885.

Martínez, G., Merinero, M., Pérez-Aranda, M., Pérez-Soriano, E.M., Ortiz, T., Begines, B., Alcudia, A., 2020. Environmental impact of nanoparticles' application as an emerging technology: a review. Materials (Basel) 14 (1), E166.

Piccino, F., Gottschalk, F., Seeger, S., Nowack, B., 2012. Industrial production quantities and uses of ten engineered nanomaterials in Europe and the world. J. Nanopart. Res. 14, 1109.

Quik, J.T., Lynch, I., Van Hoecke, K., Miermans, C.J., De Schamphelaere, K.A., Janssen, C.R., Dawson, K.A., Stuart, M.A., Van De Meent, D., 2010. Effect of natural organic matter on cerium dioxide nanoparticles settling in model fresh water. Chemosphere 81, 711-715.

Rundle, A., Robertson, A.B., Blay, A.M., Butler, K.M., Callaghan, N.I., Dieni, C.A. MacCormack, T.J., 2016. Cerium oxide nanoparticles exhibit minimal cardiac and cytotoxicity in the freshwater fish Catostomus commersonii. Comp. Biochem. Physiol. C Toxicol. Pharmacol. 181-182, 19-26.

Savolainen, K., Backman, U., Brouwer, D., Fadeel, B., Fernandes, T., Kuhlbusch, T., Landsiedel, R., Lynch, I., Pylkkänen, L., 2013. Nanosafety in Europe 2015-2025: Towards Safe and Sustainable Nanomaterials and Nanotechnology Innovations. Helsinki, Finnish Inst Occup. Heal http://www.eu-vri.eu/filehandler.ashx?file $=12392$

Singh, N., Khandelwal, N., Tiwari, E., Naskar, N., Lahiri, S., Lützenkirchen, J., Darbha, G.K., 2021. Interaction of metal oxide nanoparticles with microplastics: impact of weathering under riverine conditions. Water Res. 189, 116622.
Taylor, N.S., Merrifield, R., Williams, T.D., Chipman, J.K., Lead, J.R., Viant, M.R., 2016. Molecular toxicity of cerium oxide nanoparticles to the freshwater alga Chlamydomonas reinhardtii is associated with supra-environmental exposure concentrations. Nanotoxicology 10, 32-41.

Van Hoecke, K., De Schamphelaere, K.A., Van der Meeren, P., Smagghe, G., Janssen, C.R., 2011. Aggregation and ecotoxicity of $\mathrm{CeO}_{2}$ nanoparticles in synthetic and natural waters with variable $\mathrm{pH}$, organic matter concentration and ionic strength. Environ. Pollut. 159, 970-976

Verweij, W., Durand, A.M., Maas, J.L., van der Grinten, E., 2009. Chydotox toxicity test in toxicity measurements in concentrated water samples. RIVM Report 607013011/ 2009. National Institute of Public Health and the Environment, Bilthoven, the Netherlands.

Wang, Y., Nowack, B., 2018. Environmental risk assessment of engineered nano- $\mathrm{SiO}_{2}$, nano iron oxides, nano- $\mathrm{CeO}_{2}$, nano- $\mathrm{Al}_{2} \mathrm{O}_{3}$, and quantum dots. Environ. Toxicol. Chem. 37, 1387-1395.

Wang, Z., Chen, J., Li, X., Shao, J., Peijnenburg, W.J.G.M., 2012. Aquatic toxicity of nanosilver colloids to different trophic organisms: contributions of particles and free silver ion. Environ. Toxicol. Chem. 31, 2408-2413.

Yu, S., Liu, J., Yin, Y., Shen, M., 2018. Interactions between engineered nanoparticles and dissolved organic matter: a review on mechanisms and environmental effects. J. Environ. Sci. (China) 63, 198-217. 